\title{
Genetic Basis for Dormancy in Wild Oat
}

\author{
Michael E. Foley \\ U.S. Department of Agriculture-Agriculture Research Service, Bioscience Research Laboratory, P.O. Box 5674, \\ Fargo, ND 58105-5674
}

Wild oat (Avena fatua $\mathrm{L}$.) is a serious weed because it mimics the life cycle of small-grain crops and displays seed dormancy. Wild oat is used as an experimental system to investigate dormancy and afterripening under warm-dry conditions. Dormancy in wild oat is controlled by the action and interaction of genetic and environmental factors. In the 1970s, Canadian researchers made reciprocal crosses of some highly inbred dormant and nondormant lines of wild oat and developed a preliminary genetic model for dormancy (Jana et al., 1979). To further investigate the inheritance of dormancy, we crossed a well-characterized and highly inbred dormant (M73) with a nondormant (SH430) line of wild oat. Germinability was evaluated for seeds in an $\mathrm{F}_{2}$, backcross, and a recombinant inbred (RI) line population segregating for dormancy. The data were used to develop a genetic model for dormancy, identify molecular markers associated with quantitative trait loci (QTL) for dormancy, and investigate a genotype $\mathrm{x}$ environment interaction on germinability. Germination phenotypes were determined by imbibing all populations at $15^{\circ} \mathrm{C}$ for 6 weeks and recording the relative number of days to germination. Progeny testing was used to verify the germination phenotypes. The most dormant caryopses did not germinate. Dormancy is recessive and at least three genes regulate dormancy in wild oat. One or more of these genes is responsive to germination temperature. Genes at the $G_{1}$ and $G_{2}$ loci promote early germination, while the third gene $(D)$ delays germina-

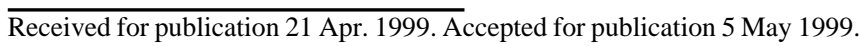
The cost of publishing this paper was defrayed in part by the payment of page charges. Under postal regulations, this paper therefore must be hereby marked advertisement solely to indicate this fact. tion. According to our model, if at least two copies of the dominant $G_{l}$ or $G_{2}$ allele are present (i.e., two at the $G_{1}$ locus, or two at the $G_{2}$ locus), regardless of the genotype at the $D$ locus, then the seed will be nondormant. If the genotype is $G_{1} g_{1} G_{2} g_{2}$, then the seed will be nondormant, provided that the genotype at the $D$ locus is $D d$ or $d d$. If only one allele of either $G_{1}$ or $G_{2}$, or none, is present and the genotype is $d d$, then the seed will also be nondormant. The $G_{1} g_{1} G_{2} g_{2} D D$, $G_{1} g_{1} g_{2} g_{2} D D, G_{1} g_{1} g_{2} g_{2} D d$, and $g_{1} g_{1} G_{2} g_{2} D d$ individuals will have a phenotype intermediate between nondormant and dormant. If the genotype is $g_{1} g_{1} G_{2} g_{2} D D$ or $g_{1} g_{1} g_{2} g_{2} D_{-}$, then the phenotype will be dormant. Using bulked segregant analysis and random amplified polymorphic DNA (RAPD) techniques, we identified two independently segregating markers that are linked in coupling with QTL for dormancy in wild oat. Further research is needed to identify additional and tightly linked molecular markers for dormancy QTL. Molecular markers will facilitate improvements in our model and genotypic classification of RI lines. Investigation of allelic interactions, epistatic interactions, and genotype $\times$ environment interactions will be essential for understanding the fundamental basis for dormancy.

An abstract only is presented here because a detailed paper was previously published (Foley and Fennimore, 1998).

\section{Literature Cited}

Foley, M.E. and S.A. Fennimore. 1998. Genetic basis for seed dormancy. Seed Sci. Res. 8:173-182.

Jana, S., N. Acharya, and J.M. Naylor. 1979. Dormancy studies in seeds of Avena fatua. 10. On the inheritance of germination behavior. Can. J. Bot. 57:1663-1667. 